\title{
Chromosomal copy number analysis on chorionic villus samples from early spontaneous miscarriages by high throughput genetic technology
}

\author{
Jiandong Shen, Wei Wu, Chao Gao, Humphrey Ochin, Dianyun Qu, Jiazi Xie, Li Gao, Yadong Zhou, Yugui Cui
} and Jiayin Liu*

\begin{abstract}
Background: About $10-15 \%$ of all clinically recognized pregnancies result in spontaneous miscarriages, and chromosomal abnormalities are the most common reason. The conventional karyotyping on chorionic villus samples (CVSs) is limited by cell culture and its resolution. This study aimed at evaluating the efficiency of the application of high throughput genetic technology, including array comparative genomic hybridization (array CGH) and next generation sequencing (NGS) on the chromosomal copy number analysis of CVSs from early spontaneous miscarriages.

Results: Four hundred and thirty-six CVSs from early spontaneous abortion were collected. Genomic DNA was extracted using a routine method, and the chromosomal copy number variants (CNVs) were analyzed by array CGH and NGS. Two hundred and twenty-five samples (51.6\%) with abnormal chromosomes were identified among 436 samples, of which 188 samples (41.3\%) were aneuploidy, 23 samples (5.3\%) were segmental deletion and/or duplication cases, and 14 samples (3.2\%) were triploid. Two of the three cases with small segmental deletion and duplication were validated to be transferred from their fathers who were carriers of submicroscopic reciprocal translocation.

Conclusion: A high chromosomal abnormality detection rate on CVSs from early spontaneous miscarriage was achieved by array CGH and NGS. Specifically, the detection of submicroscopic recombination, which is sometimes missed by conventional karyotyping, was important for genetic counseling for the couples that suffered from recurrent miscarriages.
\end{abstract}

Keywords: Array comparative genomic hybridization, Next generation sequencing, Spontaneous miscarriage, Chorionic villus samples, Chromosome

\section{Background}

Miscarriage is the most common complication of pregnancy. About $10-15 \%$ of all clinically recognized pregnancies result in spontaneous miscarriage [1]. Chromosomal abnormalities account for $\sim 45 \%$ of early spontaneous miscarriages [2]. G-banding karyotyping is a traditional method of chromosomal analysis, and it plays an important role in

\footnotetext{
* Correspondence: jyliu_nj@126.com

Department of Reproductive Medicine, The First Affiliated Hospital of Nanjing

Medical University, State Key Laboratory of Reproductive Medicine, Nanjing 210029, China
}

investigating the reason for spontaneous abortion. However, G-banding karyotyping is hampered by poor chromosome preparations, culture failure, and maternal cell contamination. Molecular karyotype approaches, such as multiplex fluorescence in situ hybridization (mFISH), multiplex ligation-dependent probe amplification (MLPA), and quantitative real-time polymerase chain reaction (qPCR), have overcome some disadvantages inherent to conventional cytogenetic techniques. However, they are criticized for their restricted resolutions and/or limited coverage on the whole genome [3-5]. In this study, we 
apply array comparative genomic hybridization (array $\mathrm{CGH}$ ) and next generation sequencing (NGS) technology to detect chromosomal abnormalities on chorionic villus samples (CVSs) from women who had early spontaneous miscarriages.

\section{Methods}

\section{Samples collection and DNA extraction}

The study was approved by Institutional Review Board of the First Affiliated Hospital of Nanjing Medical University. With informed consent, four hundred and thirty-six CVSs from women who had spontaneous miscarriages were collected, and all the miscarriages occurred between 5 to 12 weeks in gestational age. Each sample was rinsed in normal saline solution three times. Then $10 \mathrm{mg}$ of the tissue was submitted to extract genomic DNA using a DNA extraction kit (Tiangen, China).

\section{Chromosomal copy number analysis by array CGH}

Genomic DNA samples were fluorescently labelled and competitively hybridized to CytoChip Focus Constitutional microarrays (Illumina, USA) with a normal male control gDNA in an array CGH experiment format. A laser scanner InnoScan w710AL (Innopsys, France) was used to excite the hybridized fluorophores and read and store the resulting images of the hybridization. Scanned images were then analyzed and quantified by an algorithm with fixed settings in BlueFuse Multi Software (Illumina, USA) (available protocol at www.cytochip.com).

\section{Chromosomal copy number analysis by NGS and validation}

Whole genome sequencing by NGS technology was performed on an Ion torrent PGM (ThermoFisher, USA) platform according to the standard protocol (protocol available at https://ioncommunity.thermofisher.com/community/protocols-home). Genomic DNA from CVSs was sheared to 250-300 bp fragments using Ion Shear Plus Reagents Kit (ThermoFisher, USA). Ion Torrent barcoded libraries were made using Ion Plus Fragment Library Kit (ThermoFisher, USA). Ion PGM Template OT2 200 Kit (ThermoFisher, USA) was used for template amplification and enrichment of target sequence. Ion Sphere Particles (ISPs) were recovered and template-positive ISPs were enriched using an Ion OneTouch ES (ThermoFisher, USA). Sequencing was performed using an Ion PGM Sequencing 200 Kit v2 (ThermoFisher, USA) on '318' sequencing chip for a total of 500 nucleotide flows, yielding average read lengths of 220-230 bp. Ten DNA samples were pooled together and labeled with different barcodes on '318' chip. The average whole genomic sequence depth was $\sim 0.02 \times$, and the average read number was $\sim 500 \mathrm{~K}$. The primary sequencing BAM data were submitted to the Celloud cloud server (available at http://www.celloud.org/), which was offered by a third-party company (JBRH, China), in order to analyze the chromosomal copy number variants (CNVs). The pipeline of the data analysis was done according to the previous report [6]. Before using NGS to detect chromosomal CNVs routinely, validation work was performed. Ten CVSs with different types known of chromosomal abnormalities, which were confirmed by array CGH, were submitted to sequence blindly. Subsequently the consistency of the results between NGS and array CGH were analyzed.

\section{G-banding karyotyping}

Lymphocytes that were isolated from the patients were cultured and harvested after stimulation with phytohemagglutinin for $72 \mathrm{~h}$. Metaphase chromosomes were prepared according to standard cytogenetic protocols. Karyotypes were described according to the International System for Human Cytogenetic Nomenclature 2013 (ISCN 2013).

\section{Parental origin analysis of chromosomal aberration by FISH} FISH was performed on metaphase chromosomes of the lymphocytes using telomere probes (Vysis, IL) according to the previous protocol [7]. Lymphocytes were cultured and harvested after stimulation with phytohemagglutinin for $72 \mathrm{~h}$, and metaphase chromosomes were fixed on slides. After degeneration at $78{ }^{\circ} \mathrm{C}$ for $5 \mathrm{~min}$, probes with fluorescence labeling were hybridized to the chromosomes on the slides at $37{ }^{\circ} \mathrm{C}$ for $16 \mathrm{~h}$. The slides were washed in $2 \times$ SSC (Sigma, USA) and dyed with DAPI (Vysis, IL). The signals under a fluorescence microscope were observed (Leica, GER).

\section{Results}

The NGS results of the 10 samples were completely consistent with those of array CGH (see Table 1 and Fig. 1); therefore, more samples were submitted for testing using the NGS method. A total of 436 samples were tested, 256 cases of which were tested by array CGH, and 180 cases were tested by NGS. Because detection coverage was theoretically consistent based on array $\mathrm{CGH}$ and NGS, we calculated the detection results by using the two methods together. Two hundred and twenty-five cases were found to have abnormal chromosomes, which accounted for $51.6 \%$ of all the cases. There were 188 (43.1\%) cases with aneuploidy, 23 (5.3\%) cases with chromosomal segmental duplication and/or deletion, and 14 (3.2\%) cases with polyploidy (see Table 2). A total of 110 female samples and 101 male samples were found in the normal samples.

As observed in this study, aneuploidy was involved in almost all the chromosomes, except chromosome 1, and trisomy was the most common, especially in chromosome 16 . 
Table 1 Validation of copy number analysis by NGS

\begin{tabular}{|c|c|c|}
\hline NO & Results by array $\mathrm{CGH}$ & Consistency with NGS \\
\hline C0005 & $+2 ; X Y$ & Yes \\
\hline C0003 & $+16 ; X X$ & Yes \\
\hline C0015 & $+21 ; X X$ & Yes \\
\hline C0029 & $-X$ & Yes \\
\hline C0142 & +15(Mosaic); $X Y$ & Yes \\
\hline C0021 & $69, X X Y$ & Yes \\
\hline C0123 & $69, X Y Y$ & Yes \\
\hline C0012 & -(2q37.3-qter)(4.6 M), +(6q23.2-qter)(36.1 M);XX & Yes \\
\hline C0146 & +(9q34.11-qter)(9.2 M), -(14q32.13-qter)(14.8 M);XX & Yes \\
\hline C0179 & Euploid; XX & Yes \\
\hline
\end{tabular}

Monosomy was mainly found in sex chromosomes except for one case with monosomy 21 . Some aneuploidy samples involved two chromosomes. The total chromosomal numerical abnormality frequency was 198 , for all the chromosomes except chromosome 1 . Trisomy 16 was the most common in 50/198 (25.3\%), followed by chromosomes X, 22, 15, 14, and 21 (see Fig. 2).
Segmental deletion and/or duplication was found in 23/436 (5.3 \%) (see Table 3). Seventeen couples' karyotypes were available, and fifteen couples were reported as having normal karyotype, except for 2 reciprocal translocation carriers. Three couples with normal karyotype were submitted for FISH analysis, and two of them were identified to be submicroscopic

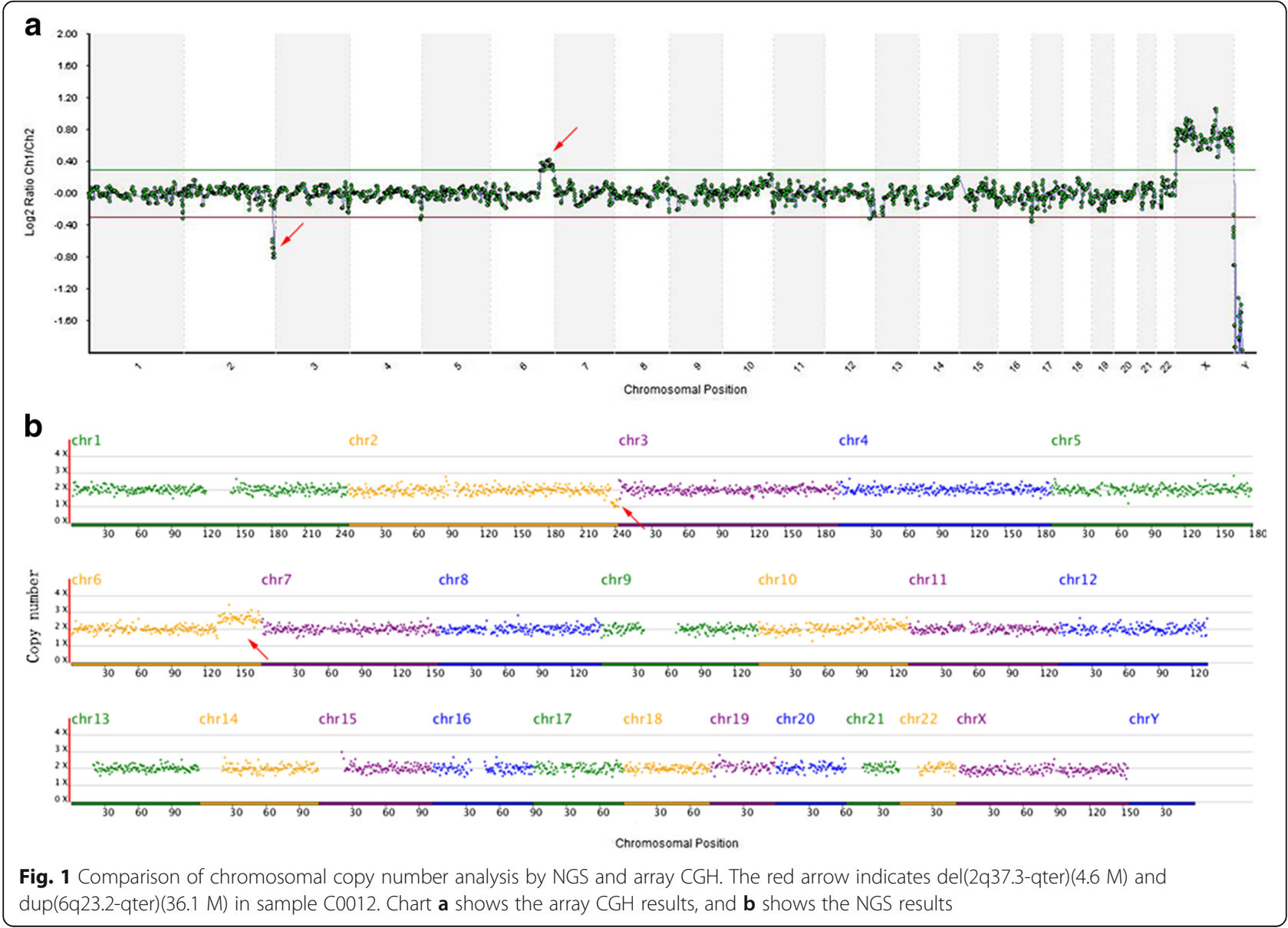


Table 2 Summary of chromosomal copy number analysis of CVSs

\begin{tabular}{llllllll}
\hline Method & Maternal age & \multicolumn{2}{l}{ Chromosomal abnormality } & \multirow{2}{*}{ Euploidy } \\
\cline { 3 - 6 } & & & Aneuploidy & Dup/Del & Polyploidy & Total & \\
\hline Array CGH & $30.8 \pm 4.3$ & $115(44.9 \%)$ & $7(2.7 \%)$ & $9(3.5 \%)$ & $131(51.2 \%)$ & $125(48.8 \%)$ & 256 \\
NGS & $31.1 \pm 4.8$ & $73(40.6 \%)$ & $16^{\mathrm{b}}(8.9 \%)$ & $5^{\mathrm{c}}(2.8 \%)$ & $94(52.2 \%)$ & $86(47.8 \%)$ & 180 \\
Total & $30.9 \pm 4.5$ & $188(43.1 \%)$ & $23(5.3 \%)$ & $14(3.2 \%)$ & $225(51.6 \%)$ & $211(48.4 \%)$ & 436 \\
\hline
\end{tabular}

array CGH and NGS could identify some polyploidy such as $69 \mathrm{XXY}$ and $69 \mathrm{XYY}$, but could not find $69 \mathrm{XXX}$, which was no sex chromosomal segregation. ${ }^{\mathrm{b}}$ two of them combined with aneuploidy; ${ }^{c}$ one of them combined with segmental duplication and tetrasomy

reciprocal balanced translocation (see Table 4 and Fig. 3).

\section{Discussion}

Chromosomal abnormality is the main reason for firsttrimester miscarriages. Conventional G-banding karyotyping is used as a golden standard method to detect chromosomal aneuploidy and imbalances. However, the overall detection failure rate was $\sim 20 \%[2,4]$, and sometimes false negative outcomes resulted from the overgrowth of maternal cells in relation to fetal cells $[5,8,9]$. Array CGH is a rapid, automated, reliable, and highresolution technique used to diagnose unbalanced chromosomal abnormalities in CVSs from miscarriage patients $[10,11]$. Recently, NGS was validated as being able to reliably detect the CNVs in CVSs [12]. Array CGH and NGS are both high throughput genetic test platforms that had revolutionary impacts on traditional cytogenetics [13-15]. We validated the efficiency of chromosomal copy number analysis through the NGS method in our lab and summarized the total detection efficiency of array CGH and NGS methods on CVSs. Here, a total number of 436 CVSs from early miscarriages were analyzed by array CGH and NGS with a
$100 \%$ diagnosis rate. We achieved a $51.6 \%$ detection rate.

The occurrence frequency of each aneuploid chromosome was analyzed in this study, which suggested that errors were involved in all chromosomes besides chromosome 1. However, limited chromosome probe panels were always used to analyze the prenatal samples [16], miscarriage samples [4, 17], and pre-implantation embryos $[18,19]$. According to this study, the limited probe panel $(\mathrm{Chr} 13,18,21, \mathrm{X}, \mathrm{Y})$ can only detect $43 / 198$ (21.7 \%) aneuploidy in CVSs, and the probe panel (Chr13, 16, 18, 21, 22, X ,Y) can only detect 115/198 (58.1\%) as well. It is obvious that a limited chromosome analysis method for CVSs is not suitable because many positive samples would be ignored. This also reflects why pre-implantation genetic screening using mFISH was proved to have no benefit for improving in vivo fertilization outcomes [20].

It is generally acknowledged that the resolution of routine G-banding karyotyping is 5-10 Mb. However, when the chromosomal segmental imbalances were involved in atypical bands, or poor digestion and dyeing were taken place in the procedure of chromosome preparation, even more than $10 \mathrm{Mb}$ segmental duplications or

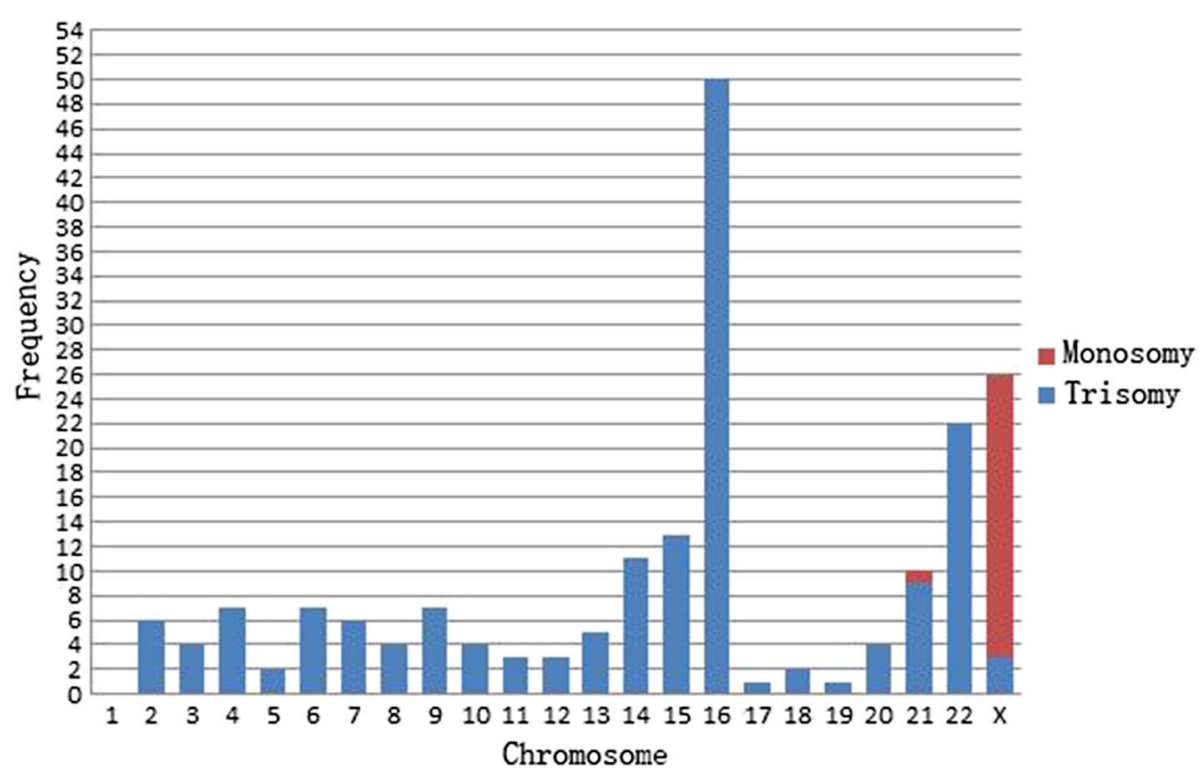

Fig. 2 The frequency of each chromosomal aneuploidy 
Table 3 Parents karyotype analysis of the CVSs with segmental copy number variants

\begin{tabular}{|c|c|c|}
\hline No & Copy number variants for CVSs & Parents karyotype \\
\hline C0012 & -(2q37.3-qter)(4.6 M); +(6q23.2-qter)(36.1 M) & Normal \\
\hline C0044 & -(6q25.3-qter)(12.5 M); +(10q26.11-qter)(13.8 M) & Normal \\
\hline C0146 & +(9q34.11-qter)(9.2 M); -(14q32.13-qter)(14.8 M) & Normal \\
\hline C0391 & -(1p36.21-pter)(12.36 M) & Normal \\
\hline C0001 & -(18p11-pter)(14.0 M); +(18q11-qter)(59.4 M) & Normal \\
\hline C0052 & +(17q21.31-qter)(38.9 M) & Normal \\
\hline C0227 & -(8p12-pter)(28.8 M); +(8q24.3-qter)(4.9 M) & Normal \\
\hline C0361 & $+(5 q$ 13.2-qter)(106.42 M); -(15q26.1-qter)(11.35 M) & Normal \\
\hline C0376 & -(5p15.1-pter)(16.6 M); +(9q21.32-qter)(56.6 M) & Normal \\
\hline C0403 & -(5p14.1-pter)(25.68 M); +(19q13.33-qter)(8.35 M) & Normal \\
\hline C0407 & $+(2 q 12.1-q 33.1)(93.02 M)$ & Normal \\
\hline C0419 & +19(q13.33-qter)(7.56 M) & Normal \\
\hline C0420 & +(11q23.3-pter)(111.15 M); -(22q11.1-q11.21)(3.92 M) & Normal \\
\hline C0432 & +(9p21.3-pter)(21.71 M); -3(q28-qter)(8.3 M) & Normal \\
\hline C0439 & -(1p36.21-pter)(13.69 M); +19 & Normal \\
\hline C0404 & +(5p13.33-pter)(31.09 M); -(10q24.32-qter)(29.19 M) & Paternal reciprocal translocation carrier \\
\hline C0193 & $+(2 p 24.3-p t e r)(15.11 \mathrm{M}) ;-(13 q 22.1$-qter)(38.47 M) & Paternal reciprocal translocation carrier \\
\hline C0063 & $+(16 p 11.2$-qter $)(56.4 \mathrm{M})$ & Loss to Follow-up \\
\hline C0195 & -(8q24.13-qter)(19.83 M); +(11q23.3-qter)(17.22 M) & Loss to Follow-up \\
\hline C0294 & +(11q22.3-q24.2)(22.08 Mb); -(1 1q24.2-qter)(7.12 Mb) & Loss to Follow-up \\
\hline C0358 & -(13q21.31-qter)(48.84 Mb) & Loss to Follow-up \\
\hline C0385 & $+14(q 11.2-q 12)(8.67 \mathrm{M}) ;-X$ & Loss to Follow-up \\
\hline C0389 & -(18p11.21-pter)(14.08 Mb); +(18p11.21-qter)(59.8 Mb); +(19q12-qter)(27.58 Mb) & Loss to Follow-up \\
\hline
\end{tabular}

deletions were sometimes missed, such as samples C0044 and C0146 (see Table 4). Array CGH had been used to identify small-size CNVs on miscarriage samples [2]; however, most of the CNVs found in those studies had no parental origin analysis and no significant clinical value was concluded. In this study, segmental deletion and/or duplication was observed in $5.3 \%$ of the samples by array $\mathrm{CGH}$ or NGS. We failed to analyze the origin of the segmental changes in all 15 of the couples with normal karyotypes. However, FISH was performed on three of them to investigate the parental origin, and two of them were proved to be hereditary from the paternal submicroscopic reciprocal balanced translocations. With exact chromosomal diagnosis, pre-implantation genetic diagnosis (PGD) was recommended to these couples [21]. Although we could not conclude the exact incidence of submicroscopic recombination in miscarriage couples with normal karyotypes, we emphasized that a clinician should be aware of submicroscopic reciprocal translocation in couples with recurrent miscarriages.

With consideration of identifying submicroscopic reciprocal translocation, high throughput genetic testing is

Table 4 Origin analysis of 3 cases with small-size segmental imbalances by FISH

\begin{tabular}{lllll}
\hline No. & Copy number variants & FISH test & & Maternal \\
\cline { 3 - 5 } & & Probe & Paternal & Parental \\
origin
\end{tabular}

${ }^{\mathrm{a}} \mathrm{FISH}$ test was performed on the metaphase of lymphocytes using telomeric probes. SG spectrum of green, SO spectrum of orange. Carrier, reciprocal balanced translocation carrier 


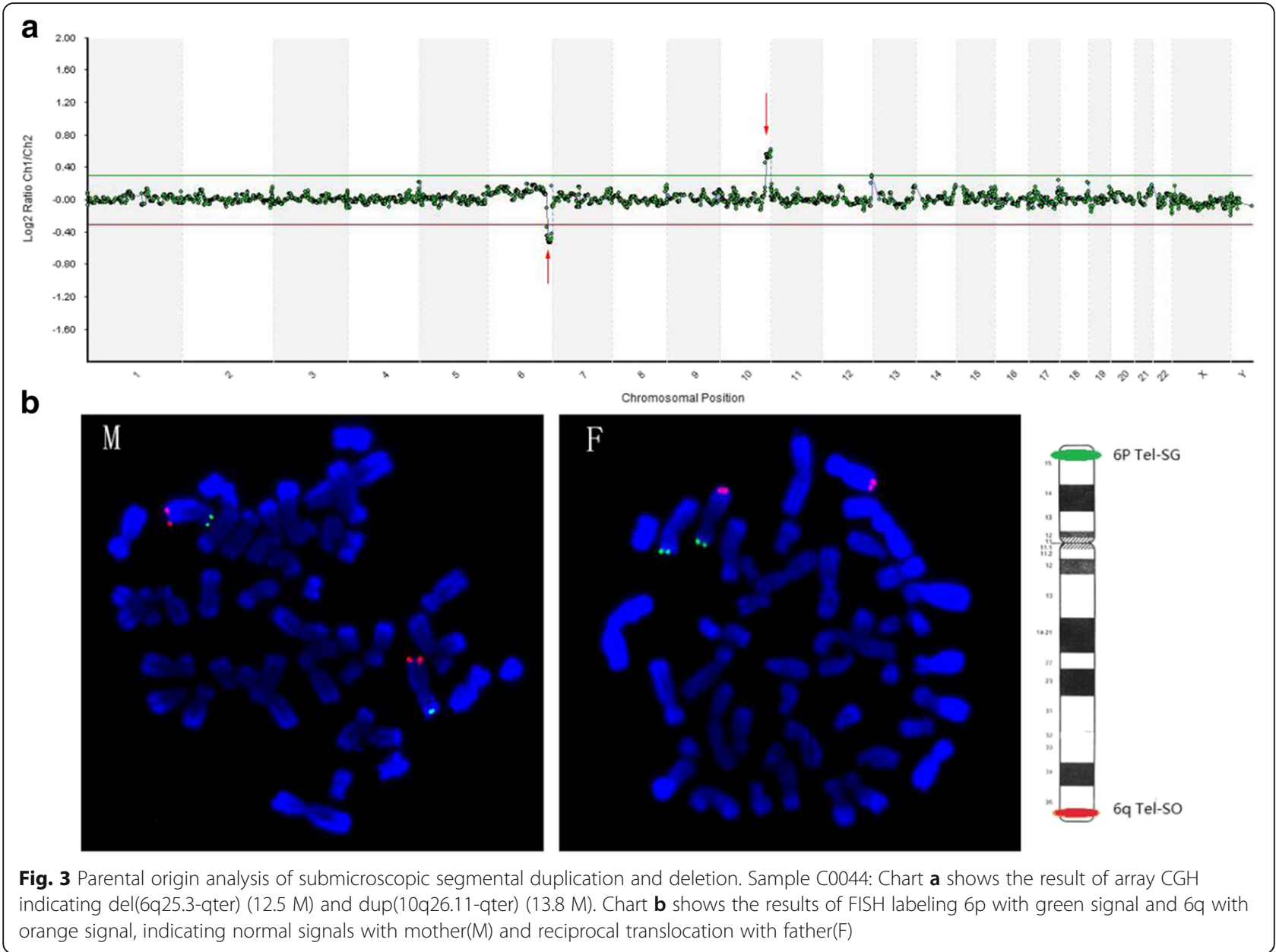

recommended for analyzing the chromosomal copy number of CVSs from spontaneous abortions. Array CGH and NGS were robust in the detection of chromosomal CNVs, and $69 \mathrm{XXY}$ and $69 \mathrm{XYY}$ could be detected as well for the special segregation of sex chromosomes. However, the limitations also should be considered. NGS and array $\mathrm{CGH}$ cannot detect all polyploidies, such as $69 \mathrm{XXX}, 92$ $\mathrm{XXXX}$, and $92 \mathrm{XXYY}$, as well as balanced translocations. In present study, more normal female samples were observed than normal male samples (110 vs 101), which was likely caused by confusion of 69 XXX and 46 XX. In the future, single nucleotide polymorphism analysis could be adopted in order to identify the polyploidies by NGS.

\section{Conclusions}

In conclusion, a high chromosomal abnormality detection rate on CVSs from patients who had spontaneous miscarriages was achieved by array CGH and NGS. Particularly, submicroscopic recombination could be detected, which was important to genetic counseling. Array CGH and NGS are comprehensive, rapid, and high-resolution chromosomal copy number analysis methods.
Competing interests

The authors declare that they have no competing interests.

\section{Authors' contributions}

JS carried out the molecular cytogenetic studies, participated in the design of the study and data analysis, and drafted the manuscript. WW carried out the genetic counseling and sample collecting. $\mathrm{HO}$ participated in genetic analysis and revising the manuscript. CG, DQ and JX participated in the array CGH and NGS and FISH essay. LG participated in sample collecting and preparing. YZ participated in G-banding karyotyping. YC participated in the design of the study. JL conceived of the study, and participated in its design and coordination and helped to draft the manuscript. All authors read and approved the final manuscript.

\section{Acknowledgement}

We would like to thank Dr. Tao Feng for helping to analyze the NGS data. This work was supported by China National 973 program (grant number 2012CB944902), Jiangsu Provincial Science and Technology Project (grant number BL20122009 and BE2011798), and the Priority Academic Program Development of Jiangsu Higher Education Institutions (PAPD).

\section{Funding information}

National Fund for Natural Science (81200444).

Received: 11 August 2015 Accepted: 21 December 2015

Published online: 26 January 2016

\section{References}

1. Rai R, Regan L. Recurrent miscarriage. Lancet. 2006;368:601-11. 
2. van den Berg MM, van Maarle MC, van Wely M, Goddijn M. Genetics of early miscarriage. Biochim Biophys Acta. 1822;2012:1951-9.

3. Deshpande M, Harper J, Holloway M, Palmer R, Wang R. Evaluation of array comparative genomic hybridization for genetic analysis of chorionic villus sampling from pregnancy loss in comparison to karyotyping and multiplex ligation-dependent probe amplification. Genet Test Mol Biomarkers. 2010; 14:421-4.

4. Shearer BM, Thorland EC, Carlson AW, Jalal SM, Ketterling RP. Reflex fluorescent in situ hybridization testing for unsuccessful product of conception cultures: a retrospective analysis of 5555 samples attempted by conventional cytogenetics and fluorescent in situ hybridization. Genet Med. 2011;13:545-52.

5. Diego-Alvarez D, Garcia-Hoyos M, Trujillo MJ, Gonzalez-Gonzalez C, Rodriguez de Alba M, Ayuso C, et al. Application of quantitative fluorescent PCR with short tandem repeat markers to the study of aneuploidies in spontaneous miscarriages. Hum Reprod. 2005;20:1235-43.

6. Hou Y, Fan W, Yan L, Li R, Lian Y, Huang J, et al. Genome analyses of single human oocytes. Cell. 2013;155:1492-506.

7. Shen JD, Liang DS, Zhou ZM, Xia Y, Long ZG, Wu LQ. Pallister-Killian syndrome: meiosis II non-disjunction may be the first step in the formation of isochromosome 12p. Chin Med J (Engl). 2010;123:3482-5.

8. Robberecht C, Schuddinck V, Fryns JP, Vermeesch JR. Diagnosis of miscarriages by molecular karyotyping: benefits and pitfalls. Genet Med. 2009;11:646-54.

9. Karaoguz MY, Nas T, Konac E, Ince D, Pala E, Menevse S. Is cytogenetic diagnosis of 46, XX karyotype spontaneous abortion specimens erroneous? Fluorescence in situ hybridization as a confirmatory technique. J Obstet Gynaecol Res. 2005;31:508-13.

10. Menten B, Swerts K, Delle Chiaie B, Janssens S, Buysse K, Philippe J, et al. Array comparative genomic hybridization and flow cytometry analysis of spontaneous abortions and mors in utero samples. BMC Med Genet. 2009;10:89.

11. Schaeffer AJ, Chung J, Heretis K, Wong A, Ledbetter DH, Lese Martin C. Comparative genomic hybridization-array analysis enhances the detection of aneuploidies and submicroscopic imbalances in spontaneous miscarriages. Am J Hum Genet. 2004;74:1168-74.

12. Xie W, Tan Y, Li X, Lin G, Jiang H, Chen F, et al. Rapid detection of aneuploidies on a benchtop sequencing platform. Prenat Diagn. 2013;33:232-7.

13. Miller DT, Adam MP, Aradhya S, Biesecker LG, Brothman AR, Carter NP, et al. Consensus statement: chromosomal microarray is a first-tier clinical diagnostic test for individuals with developmental disabilities or congenital anomalies. Am J Hum Genet. 2010;86:749-64.

14. Biesecker LG, Green RC. Diagnostic clinical genome and exome sequencing. N Engl J Med. 2014;370:2418-25.

15. Koboldt DC, Steinberg KM, Larson DE, Wilson RK, Mardis ER. The nextgeneration sequencing revolution and its impact on genomics. Cell. 2013; 155:27-38.

16. Tepperberg J, Pettenati MJ, Rao PN, Lese CM, Rita D, Wyandt H, et al. Prenatal diagnosis using interphase fluorescence in situ hybridization (FISH): 2-year multi-center retrospective study and review of the literature. Prenat Diagn. 2001;21:293-301.

17. Jobanputra V, Esteves C, Sobrino A, Brown S, Kline J, Warburton D. Using FISH to increase the yield and accuracy of karyotypes from spontaneous abortion specimens. Prenat Diagn. 2011;31:755-9.

18. Staessen C, Verpoest W, Donoso P, Haentjens P, Van der Elst J, Liebaers I, et al. Preimplantation genetic screening does not improve delivery rate in women under the age of 36 following single-embryo transfer. Hum Reprod. 2008:23:2818-25.

19. Debrock S, Melotte C, Spiessens C, Peeraer K, Vanneste E, Meeuwis L, et al. Preimplantation genetic screening for aneuploidy of embryos after in vitro fertilization in women aged at least 35 years: a prospective randomized trial. Fertil Steril. 2010;93:364-73.

20. Mastenbroek S, Twisk M, van der Veen F, Repping S. Preimplantation genetic screening: a systematic review and meta-analysis of RCTs. Hum Reprod Update. 2011;17:454-66.

21. Fiorentino F, Spizzichino L, Bono S, Biricik A, Kokkali G, Rienzi L, et al. PGD for reciprocal and Robertsonian translocations using array comparative genomic hybridization. Hum Reprod. 2011;26:1925-35.

\section{Submit your next manuscript to BioMed Central and we will help you at every step:}

- We accept pre-submission inquiries

- Our selector tool helps you to find the most relevant journal

- We provide round the clock customer support

- Convenient online submission

- Thorough peer review

- Inclusion in PubMed and all major indexing services

- Maximum visibility for your research

Submit your manuscript at www.biomedcentral.com/submit

Biomed Central 